\title{
Line narrowing of terahertz vibrational modes for organic thin polycrystalline films within a parallel plate waveguide
}

\author{
Joseph S. Melinger ${ }^{\mathrm{a})}$ \\ Naval Research Laboratory, Electronics Science and Technology Division, Code 6812, Washington, DC \\ 20375 \\ N. Laman, S. Sree Harsha, and D. Grischkowsky ${ }^{\text {b) }}$ \\ School of Electrical and Computer Engineering, Oklahoma State University, Stillwater, Oklahoma 74078
}

(Received 12 September 2006; accepted 9 November 2006; published online 19 December 2006)

\begin{abstract}
Waveguide terahertz time-domain spectroscopy is used to demonstrate the narrowing of vibrational lines for a thin polycrystalline film of 1,2-dicyanobenzene in a parallel plate metal waveguide. When compared to corresponding linewidths for 1,2-dicyanobenzene in a pellet sample at room temperature, the linewidths for the waveguide film are found to be significantly sharper. For measurements near $77 \mathrm{~K}$, a dramatic line narrowing is observed for the waveguide film, yielding linewidths as much as five times sharper than found in the pellet. These effects are attributed to much smaller inhomogeneous broadening in the waveguide film and result in a more informative spectrum. (C) 2006 American Institute of Physics. [DOI: 10.1063/1.2405884]
\end{abstract}

A standard method for measuring terahertz $(\mathrm{THz})$ spectra of organic solids is to form a pellet that contains a small quantity of the target solid dispersed in a much larger quantity of a low absorbance host material such as polyethylene. A wide range of organic and bio-organic materials in the form of such pellets has been studied in the $\mathrm{THz}$ region, including explosives materials ${ }^{1}$ and their by-products, ${ }^{2}$ conjugated oligomers, ${ }^{3}$ amino acids, ${ }^{4}$ and DNA. While much spectroscopic information can be obtained from pellet samples, in many cases their $\mathrm{THz}$ spectra exhibit broad line shapes due to intrinsic line broadening mechanisms, and to effects related to pellet preparation. ${ }^{6}$ A promising method for investigating the $\mathrm{THz}$ properties of organic solids is through the use of THz waveguides. ${ }^{7-9}$ For waveguide terahertz timedomain spectroscopy (THz-TDS) the parallel plate metal waveguide (PPWG) is particularly useful because of the nondispersive and low loss propagation for the transverse electromagnetic mode. ${ }^{7,9}$ The high detection sensitivity that is possible with the PPWG has recently been demonstrated by the THz-TDS characterization of nanometer thick water layers. ${ }^{9}$ In addition, the PPWG is easily opened, and the exposed flat inner surfaces are ideal for coating with a thin film of the investigated material.

In this letter we demonstrate the application of a PPWG to the terahertz TDS characterization of a solid organic thin polycrystalline film. The material 1,2-dicyanobenzene (12DCB) is studied because of its relatively strong vibrational lines in the $\mathrm{THz}$ region. ${ }^{10}$ The major finding of this study is that the THz spectrum of a waveguide polycrystalline film can display significantly sharper vibrational linewidths than found in the corresponding $\mathrm{THz}$ spectrum of the pellet. For measurements performed near $77 \mathrm{~K}$, a dramatic line narrowing was observed for the waveguide film, yielding linewidths as much as five times sharper than found in the pellet sample. The line narrowing effect revealed additional vibrational structure in the waveguide film which is not apparent in the pellet. We attribute the line narrowing to

\footnotetext{
${ }^{a)}$ Electronic mail: melinger@ccf.nrl.navy.mi

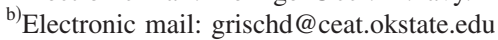

a reduction of inhomogeneous broadening for the 12DCB polycrystalline film drop cast on the metal waveguide surface. We also demonstrate the high sensitivity of the waveguide technique made possible by the subwavelength gap and long propagation length.

A schematic of the experimental apparatus for waveguide THz-TDS (Refs. 7-9) is shown in Fig. 1. The PPWG assembly consists of two copper plates with identical dimensions of $27.9 \mathrm{~mm}$ (width) $\times 30.5 \mathrm{~mm}$ (length) $\times 9.5 \mathrm{~mm}$ (thickness). A plate separation of $50 \mu \mathrm{m}$ is achieved by four $50 \mu \mathrm{m}$ thick spacers. A free-space $\mathrm{THz}$ beam is coupled into and out of the waveguide using two identical planocylindrical Si lenses at the input and exit faces of the PPWG. For experiments at low temperature the waveguide assembly is placed inside a vacuum chamber with straight through optical access and mechanically attached to the surface of a liquid nitrogen container as shown in Fig. 1.

Thin polycrystalline films of $12 \mathrm{DCB}$ (Aldrich) were made by drop casting from a $2 \mathrm{mg} / \mathrm{ml}$ solution of 12DCB in toluene onto the inner surface of one plate of an opened PPWG. Following evaporation, the relatively thick areas of the film along the perimeter were removed using a solventsoaked swab to leave a visually uniform film that was approximately $15 \mathrm{~mm}$ wide $\times 25 \mathrm{~mm}$ in length. The mass of the film was estimated by dissolving a similarly prepared

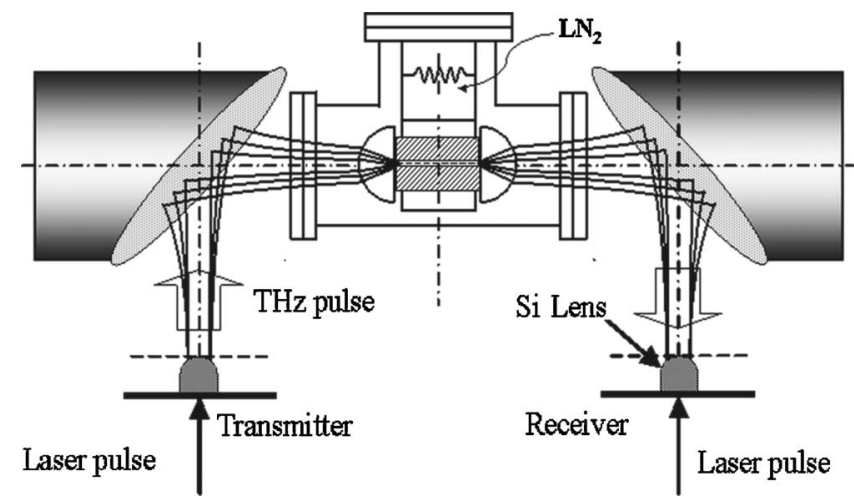

FIG. 1. Experimental setup for waveguide terahertz-TDS. 


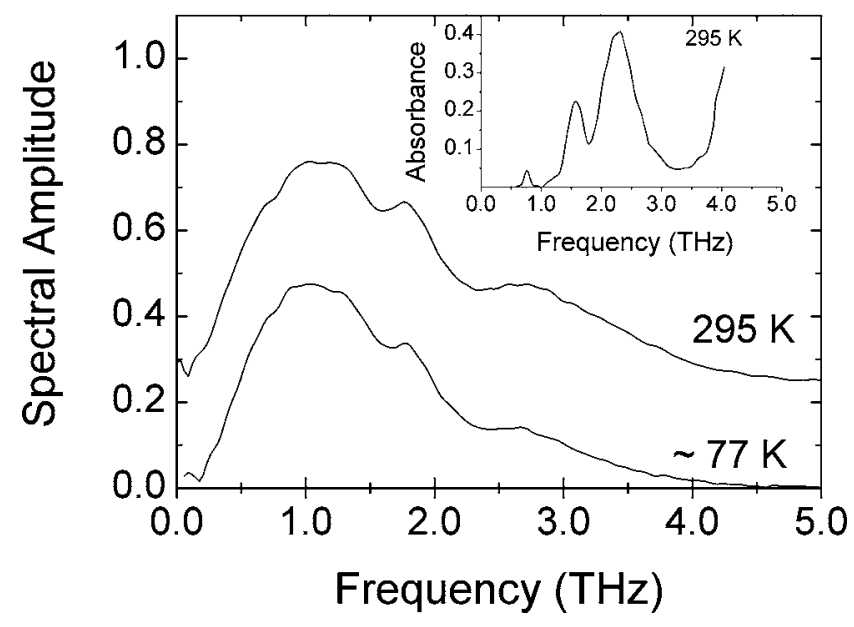

FIG. 2. THz transmission spectra for the 12DCB pellet at $295 \mathrm{~K}$ (upper curve, lifted by 0.25 for visual clarity) and $\sim 77 \mathrm{~K}$ (lower). Inset: $\mathrm{THz}$ absorbance spectrum of the 12DCB pellet at $295 \mathrm{~K}$.

film into a solution of chloroform and comparing the UV absorption spectrum to that of a 12DCB solution with a known concentration. A typical film mass was approximately $50-60 \mu \mathrm{g}$. Pellet samples were made by mixing $4.8 \mathrm{mg}$ of 12DCB powder in $100 \mathrm{mg}$ of polyethylene powder, and then pressing the mixture to yield a $1 \mathrm{~cm}$ diameter pellet with a thickness on the order of $1 \mathrm{~mm}$.

Figures 2 and 3 show $\mathrm{THz}$ spectra of the pellet and waveguide samples, respectively, at room temperature $(295 \mathrm{~K})$ and at liquid nitrogen temperature near $77 \mathrm{~K}$. The spectra are obtained by Fourier transformation of the timedomain wave forms. The mode frequencies between 0.5 and 3.0 $\mathrm{THz}$, and the observed frequency shifts with cooling to

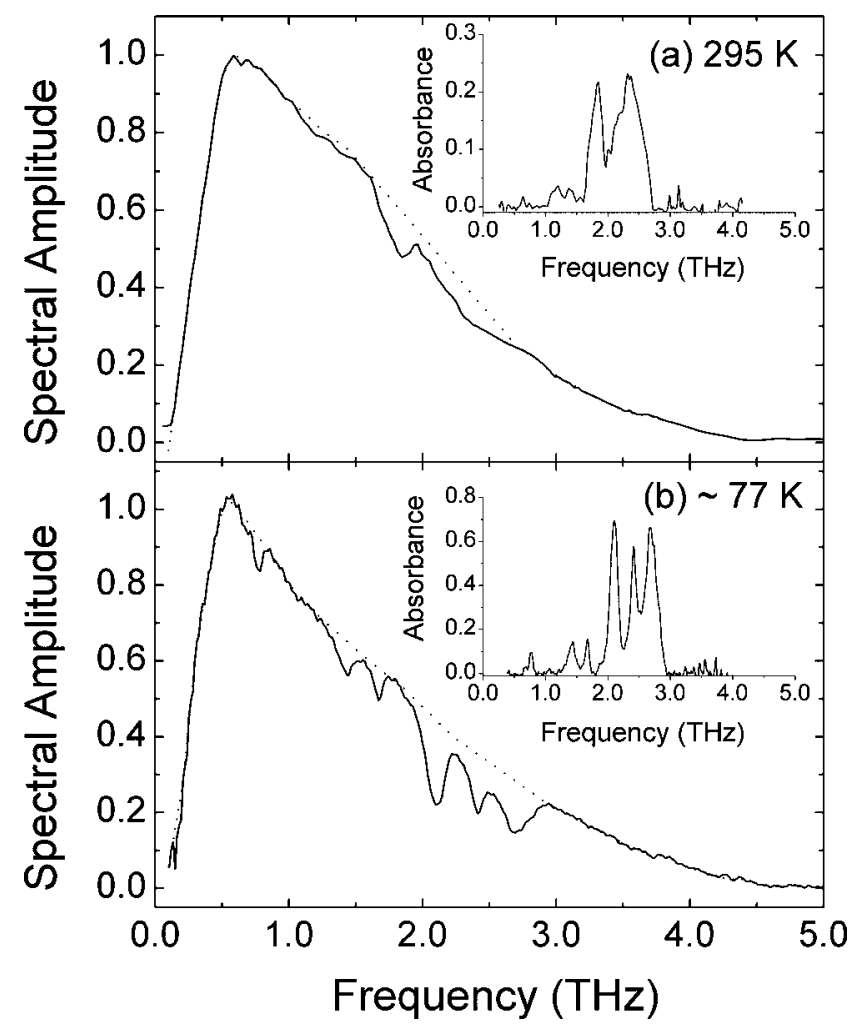

FIG. 3. THz spectra of the 12DCB polycrystalline film in the $50 \mu \mathrm{m}$ gap waveguide at (a) $295 \mathrm{~K}$ and (b) $\sim 77 \mathrm{~K}$. The insets show estimated absor-

bance spectra.
Downloaded 31 Jan 2007 to 139.78.79.178. Redistribution subject to AlP license or copyright, see http://apl.aip.org/apl/copyright.jsp
TABLE I. Mode frequencies $\nu$ in $\mathrm{THz}$ at $295 \mathrm{~K}$ and $\sim 77 \mathrm{~K}$ and frequency shifts $\Delta \nu$ in $\mathrm{THz}$ upon cooling to $\sim 77 \mathrm{~K}$.

\begin{tabular}{llccl}
\hline \hline \multirow{3}{*}{ Pellet } & Mode & $\nu_{295 \mathrm{~K}}$ & $\nu_{\sim 77 \mathrm{~K}}$ & \multicolumn{1}{c}{$\Delta \nu$} \\
\hline \multirow{6}{*}{ Waveguide } & $\mathrm{a}$ & 0.76 & 0.76 & 0.0 \\
& $\mathrm{~b}$ & 1.58 & 1.64 & 0.06 \\
& $\mathrm{c}$ & 2.29 & 2.46 & 0.17 \\
& $\mathrm{a}$ & 0.65 & 0.77 & 0.12 \\
& $\mathrm{~b}$ & 1.20 & 1.44 & 0.24 \\
& $\mathrm{~b}^{\prime}$ & 1.40 & 1.67 & 0.27 \\
& $\mathrm{~b}^{\prime \prime}$ & 1.85 & 2.10 & 0.25 \\
& $\mathrm{c}^{\prime}$ & 2.15 & 2.40 & 0.25 \\
& $\mathrm{c}^{\prime \prime}$ & 2.35 & 2.68 & 0.33 \\
\hline \hline
\end{tabular}

near $77 \mathrm{~K}$, are shown in Table I. The absorbance spectrum in the inset of Fig. 2 is obtained by normalization to a reference pellet of pure polyethylene and plotting $-\ln \left|A_{s}(\omega) / A_{r}(\omega)\right|$, where $A_{s}(\omega)$ and $A_{r}(\omega)$ are the Fourier transform transmission amplitudes of the signal and reference $\mathrm{THz}$ pulses, respectively.

The transmission spectra in Fig. 2 show the presence of three distinct lines ( $\mathrm{a}, \mathrm{b}$, and $\mathrm{c}$ in Table $\mathrm{I})$. At room temperature, the spectral peaks occur at $0.76,1.58$, and $2.29 \mathrm{THz}$, with half widths of $0.06 \mathrm{THz}\left(2 \mathrm{~cm}^{-1}\right), 0.18 \mathrm{THz}\left(5.9 \mathrm{~cm}^{-1}\right)$, and $0.35 \mathrm{THz}\left(11.6 \mathrm{~cm}^{-1}\right)$, respectively. Our results are consistent with a previous room temperature measurement of a 12DCB pellet, where the features observed below $3.0 \mathrm{THz}$ are attributed to external modes of the lattice. ${ }^{10}$ Upon cooling to near $77 \mathrm{~K}$ it may be anticipated that significant line narrowing will occur due to the slowing of dynamical relaxation processes. However, the 12DCB transmission spectrum shows only a minor narrowing of the absorption features, which is accompanied by a blue shift for the modes at 1.58 and $2.29 \mathrm{THz}$.

Figure 3 shows $\mathrm{THz}$ transmission spectra for the 12DCB film inside the PPWG. Recovering precise reference spectra was somewhat difficult because of variations in the spectral transmission upon reassembly of the PPWG consisting of a drop-cast covered plate together with a bare waveguide plate. Instead, we estimated the reference spectrum by choosing points along the transmission profile that lie outside the absorption lines and then fitting the points with a spline function to obtain a smooth reference profile, as shown by the dashed line. Absorbance spectra estimated in this manner are shown in the insets of Fig. 3. A comparison to the pellet spectra shows some striking differences. At room temperature the waveguide film reveals as many as four distinct spectral lines below $3.0 \mathrm{THz}$, and a shoulder at $2.15 \mathrm{THz}$. There is also evidence for a line beyond $4.2 \mathrm{THz}$, although the $\mathrm{THz}$ signal in this region is very low. As an experimental check, we repeated the experiment for a similar film in a longer $6.25 \mathrm{~cm}$ path length waveguide and found $\mathrm{THz}$ lines with essentially the same frequencies, and with an increase in absorption proportional to the path length increase. We estimate that the room temperature lines of the waveguide film are approximately a factor of 2 narrower than the corresponding pellet lines.

An important distinction between waveguide and pellet measurements is observed for measurements near $77 \mathrm{~K}$. Compared to the pellet, for which the spectral lines become only somewhat sharper and stronger, the spectral lines of the 
waveguide film dramatically sharpen, split, and shift to higher frequency. The waveguide film clearly shows six lines below $3.0 \mathrm{THz}$ at 0.77 (a), $1.44\left(\mathrm{~b}^{\prime}\right), 1.67\left(\mathrm{~b}^{\prime \prime}\right), 2.10\left(\mathrm{c}^{\prime}\right)$, $2.40\left(\mathrm{c}^{\prime \prime}\right)$, and $2.68\left(\mathrm{c}^{\prime \prime \prime}\right)$ THz. The narrowest lines at 1.67 and $2.10 \mathrm{THz}$ have half widths of approximately $0.025 \mathrm{THz}$ $\left(0.83 \mathrm{~cm}^{-1}\right)$ and $0.065 \mathrm{THz}\left(2.2 \mathrm{~cm}^{-1}\right)$, respectively, and are more than a factor of 5 sharper than the corresponding lines (b and c) in the pellet. Furthermore, the line frequencies at low temperature are substantially blueshifted from their positions at room temperature. For example, the mode at $2.68 \mathrm{THz}$ has shifted by $0.33 \mathrm{THz}\left(\sim 11 \mathrm{~cm}^{-1}\right)$ from its room temperature position. A final distinction is that the waveguide film and pellet spectra show somewhat different relative line intensities. Most notable is the doublet $\left(b^{\prime}\right.$ and $\left.b^{\prime \prime}\right)$ in the waveguide film, which is approximately a factor of 2 smaller in relative intensity than its pellet counterpart (b).

The THz vibrational line shape reflects a convolution of homogeneous (dynamic) and inhomogeneous (static) line broadening mechanisms. Homogeneous broadening typically shows distinct temperature dependence and becomes quenched at sufficiently low temperature, ${ }^{11}$ while static inhomogeneous broadening is relatively insensitive to temperature. For the 12DCB pellet, the minor temperature dependence indicates that the vibrational line shapes are dominated by inhomogeneous broadening. A reasonable explanation is that the pellet contains a static distribution of microcrystal sizes that are randomly oriented within the polyethylene host, giving rise to strongly inhomogeneous broadening of lines. In contrast, the significant line narrowing upon cooling indicates that the lines of the waveguide film are dominated by a homogeneous broadening mechanism, which may be expected if the drop-cast polycrystalline waveguide film has a high degree of orientation on the copper waveguide surface. We note that other planar organic molecules tend to form oriented thin films on substrates. ${ }^{12}$ A microscopic inspection of the drop-cast polycrystalline film showed that it was composed of microcrystals randomly distributed over the waveguide surface, but showing significant planar orientation with respect to the surface. We believe that such order (together with the formation of low defect microcrystals) is a requirement to observe the line-narrowing effect. In fact, for sample films with no such order, the line-narrowing effect is not observed. If the narrowed linewidths of the 12DCB film near $77 \mathrm{~K}$ contain residual homogeneous broadening then further line narrowing would be expected at lower temperatures.

Upon cooling, the THz modes are observed to shift to higher frequencies for both the pellet sample and the waveguide film, although the magnitude of the blueshift is typically larger for the waveguide film. The frequency shift is, in part, explained by a compression of the crystalline lattice upon cooling, which results in a steeper intermolecular potential. Anharmonicity of the vibrational modes may also contribute to the shift. The reason for the larger shift in the waveguide film is unclear, but may be related to differences in the thermal expansion coefficients of the copper plate and the dielectric film.

For our experimental conditions, a substantial sensitivity enhancement is predicted for waveguide detection of a thin film relative to the traditional single pass through the same film thickness. For a PPWG of length $L$ and a plate separation of $b$, the sensitivity enhancement is given by $\Gamma$ $=L /\left(n_{l}^{3} b\right)$, where $n_{l}$ is the refractive index of the film in the waveguide. ${ }^{7}$ If we take $n_{l}=1.7$, which is consistent with values for other organic solids in the $\mathrm{THz}$ region, ${ }^{2}$ and using $b=50 \mu \mathrm{m}$ and $L=2.5 \mathrm{~cm}$, then $\Gamma=102$. A similar sensitivity enhancement appears to be confirmed in the experiment by the nearly two orders of magnitude smaller quantity of 12DCB in the film compared to the pellet for the similar absorption strengths observed for the pellet and waveguide samples.

In summary, our major finding is that the $\mathrm{THz}$ linewidths for a solid organic thin polycrystalline film in a PPWG can be significantly narrower than the same organic solid dispersed in a pellet. For 12DCB, the sharper linewidths in the film reveal additional vibrational structure, which is concealed in the corresponding pellet by strong inhomogeneous line broadening effects. We have made similar observations comparing waveguide films and pellets of the isomers 1,3dicyanobenzene and 1,4-dicyanobenzene. In addition, we have observed strong line narrowing for drop-cast polycrystalline films of 7,7,8,8-tetracyanoquinodimethane (TCNQ) and for the deoxynucleoside, 2-deoxycytidine. These will be reported in a future work. The additional vibrational structure found in the waveguide films will be important for advancing the understanding of the far-infrared vibrational properties of molecular solids. The increased resolution and high sensitivity of waveguide THz-TDS with PPWGs may find practical applications, such as the sensing of trace quantities of solid materials through the detection of their vibrational fingerprints.

This work was supported by the Office of Naval Research and by the National Science Foundation.

${ }^{1}$ M. C. Kemp, P. F. Taday, B. E. Cole, J. A. Cluff, A. J. Fitzgerald, and W. R. Tribe, Proc. SPIE 5070, 44 (2003).

${ }^{2}$ Y. Chen, H. Liu, Y. Deng, D. Schauki, M. J. Fitch, R. Osiander, C. Dodson, J. B. Spicer, M. Shur, and X.-C. Zhang, Chem. Phys. Lett. 400, 357 (2004).

${ }^{3}$ M. D. Johnston, L. M. Herz, A. L. T. Khan, A. Kohler, A. G. Davies, and E. H. Linfield, Chem. Phys. Lett. 377, 256 (2003).

${ }^{4}$ T. M. Korter, R. Balu, M. B. Campbell, M. C. Beard, S. K. Gregurick, and E. J. Heilweil, Chem. Phys. Lett. 418, 65 (2006).

${ }^{5}$ A. G. Markelz, A. Roitberg, and E. J. Heilweil, Chem. Phys. Lett. 320, 42 (2000).

${ }^{6}$ J. F. Federici, B. Schulkin, F. Huang, D. Gary, R. Barat, F. Oliveira, and D. Zimdars, Semicond. Sci. Technol. 20, S266 (2005).

${ }^{7}$ G. Gallot, S. P. Jamison, R. W. McGowan, and D. Grischkowsky, J. Opt. Soc. Am. B 17, 851 (2000).

${ }^{8}$ R. Mendis and D. Grischkowsky, Opt. Lett. 26, 846 (2001).

${ }^{9}$ J. Zhang and D. Grischkowsky, Opt. Lett. 29, 1617 (2004).

${ }^{10}$ O. Esenturk and E. J. Heilweil (submitted).

${ }^{11}$ D. Dlott, Annu. Rev. Phys. Chem. 37, 157 (1986).

${ }^{12}$ J. G. Laquindanum, H. E. Katz, A. J. Lovinger, and A. Dodabalapur, Chem. Mater. 8, 2542 (1996) 